$\mathrm{J}$ o u r n a l of

Mathematics

and Applications

JMA No 43, pp 139-152 (2020)

\title{
Analogy of Classical and Dynamic Inequalities Merging on Time Scales
}

\section{Muhammad Jibril Shahab Sahir}

\begin{abstract}
In this paper, we present analogues of Radon's inequality and Nesbitt's inequality on time scales. Furthermore, we find refinements of some classical inequalities such as Bergström's inequality, the weighted power mean inequality, Cauchy-Schwarz's inequality and Hölder's inequality. Our investigations unify and extend some continuous inequalities and their corresponding discrete analogues.
\end{abstract}

AMS Subject Classification: 26D15, 26D20, 34N05.

Keywords and Phrases: Time scales; Radon's inequality; The weighted power mean inequality; Hölder's inequality; Nesbitt's inequality.

\section{Introduction}

We present here some well-known classical inequalities.

If $n \in \mathbb{N}, x_{k} \geq 0$ and $y_{k}>0$ for $k \in\{1,2, \ldots, n\}$ and $\beta \geq 2$, then

$$
n^{2-\beta} \frac{\left(\sum_{k=1}^{n} x_{k}\right)^{\beta}}{\sum_{k=1}^{n} y_{k}} \leq \sum_{k=1}^{n} \frac{x_{k}^{\beta}}{y_{k}} .
$$

Inequality (1.1) is called Radon's inequality as given in [21, 22, 23, 24].

The weighted power mean inequality given in [9, pp. 111-112, Theorem 10.5], [11, pp. 12-15] and [15] is defined as follows:

Let $x_{1}, x_{2}, \ldots, x_{n}$ be nonnegative real numbers and $p_{1}, p_{2}, \ldots, p_{n}$ be positive real numbers. If $\eta_{2}>\eta_{1}>0$, then

$$
\left(\frac{p_{1} x_{1}^{\eta_{1}}+p_{2} x_{2}^{\eta_{1}}+\ldots+p_{n} x_{n}^{\eta_{1}}}{p_{1}+p_{2}+\ldots+p_{n}}\right)^{\frac{1}{\eta_{1}}} \leq\left(\frac{p_{1} x_{1}^{\eta_{2}}+p_{2} x_{2}^{\eta_{2}}+\ldots+p_{n} x_{n}^{\eta_{2}}}{p_{1}+p_{2}+\ldots+p_{n}}\right)^{\frac{1}{\eta_{2}}}
$$

COPYRIGHT (C) by Publishing House of Rzeszów University of Technology P.O. Box 85, 35-959 Rzeszów, Poland 
If $x_{k}$ and $y_{k}$ for $k \in\{1,2, \ldots, n\}$ are sequences of real numbers, then CauchySchwarz's inequality is given by:

$$
\sum_{k=1}^{n} x_{k} y_{k} \leq\left(\sum_{k=1}^{n} x_{k}^{2}\right)^{\frac{1}{2}}\left(\sum_{k=1}^{n} y_{k}^{2}\right)^{\frac{1}{2}}
$$

as given in [9].

We will prove these results on time scales. The calculus of time scales was initiated by Stefan Hilger as given in [12]. A time scale is an arbitrary nonempty closed subset of the real numbers. The theory of time scales is applied to combine results in one comprehensive form. The three most popular examples of calculus on time scales are differential calculus, difference calculus, and quantum calculus, i.e., when $\mathbb{T}=\mathbb{R}$, $\mathbb{T}=\mathbb{N}$ and $\mathbb{T}=q^{\mathbb{N}_{0}}=\left\{q^{t}: t \in \mathbb{N}_{0}\right\}$ where $q>1$. The time scales calculus is studied as delta calculus, nabla calculus and diamond- $\alpha$ calculus. This hybrid theory is also widely applied on dynamic inequalities. The basic work on dynamic inequalities is done by Ravi Agarwal, George Anastassiou, Martin Bohner, Allan Peterson, Donal O'Regan, Samir Saker and many other authors.

In this paper, it is assumed that all considerable integrals exist and are finite and $\mathbb{T}$ is a time scale, $a, b \in \mathbb{T}$ with $a<b$ and an interval $[a, b]_{\mathbb{T}}$ means the intersection of a real interval with the given time scale.

\section{Preliminaries}

We need here basic concepts of delta calculus. The results of delta calculus are adopted from monographs $[6,7]$.

For $t \in \mathbb{T}$, the forward jump operator $\sigma: \mathbb{T} \rightarrow \mathbb{T}$ is defined by

$$
\sigma(t):=\inf \{s \in \mathbb{T}: s>t\} .
$$

The mapping $\mu: \mathbb{T} \rightarrow \mathbb{R}_{0}^{+}=[0,+\infty)$ such that $\mu(t):=\sigma(t)-t$ is called the forward graininess function. The backward jump operator $\rho: \mathbb{T} \rightarrow \mathbb{T}$ is defined by

$$
\rho(t):=\sup \{s \in \mathbb{T}: s<t\} .
$$

The mapping $\nu: \mathbb{T} \rightarrow \mathbb{R}_{0}^{+}=[0,+\infty)$ such that $\nu(t):=t-\rho(t)$ is called the backward graininess function. If $\sigma(t)>t$, we say that $t$ is right-scattered, while if $\rho(t)<t$, we say that $t$ is left-scattered. Also, if $t<\sup \mathbb{T}$ and $\sigma(t)=t$, then $t$ is called right-dense, and if $t>\inf \mathbb{T}$ and $\rho(t)=t$, then $t$ is called left-dense. If $\mathbb{T}$ has a left-scattered maximum $M$, then $\mathbb{T}^{k}=\mathbb{T}-\{M\}$, otherwise $\mathbb{T}^{k}=\mathbb{T}$.

For a function $f: \mathbb{T} \rightarrow \mathbb{R}$, the delta derivative $f^{\Delta}$ is defined as follows:

Let $t \in \mathbb{T}^{k}$. If there exists $f^{\Delta}(t) \in \mathbb{R}$ such that for all $\epsilon>0$, there is a neighborhood $U$ of $t$, such that

$$
\left|f(\sigma(t))-f(s)-f^{\Delta}(t)(\sigma(t)-s)\right| \leq \epsilon|\sigma(t)-s|,
$$


for all $s \in U$, then $f$ is said to be delta differentiable at $t$, and $f^{\Delta}(t)$ is called the delta derivative of $f$ at $t$.

A function $f: \mathbb{T} \rightarrow \mathbb{R}$ is said to be right-dense continuous ( $r d$-continuous), if it is continuous at each right-dense point and there exists a finite left-sided limit at every left-dense point. The set of all rd-continuous functions is denoted by $C_{r d}(\mathbb{T}, \mathbb{R})$.

The next definition is given in $[6,7]$.

Definition 2.1. A function $F: \mathbb{T} \rightarrow \mathbb{R}$ is called a delta antiderivative of $f: \mathbb{T} \rightarrow \mathbb{R}$, provided that $F^{\Delta}(t)=f(t)$ holds for all $t \in \mathbb{T}^{k}$. Then the delta integral of $f$ is defined by

$$
\int_{a}^{b} f(t) \Delta t=F(b)-F(a) .
$$

The following results of nabla calculus are taken from $[2,6,7]$.

If $\mathbb{T}$ has a right-scattered minimum $m$, then $\mathbb{T}_{k}=\mathbb{T}-\{m\}$, otherwise $\mathbb{T}_{k}=\mathbb{T}$. A function $f: \mathbb{T}_{k} \rightarrow \mathbb{R}$ is called nabla differentiable at $t \in \mathbb{T}_{k}$, with nabla derivative $f^{\nabla}(t)$, if there exists $f^{\nabla}(t) \in \mathbb{R}$ such that given any $\epsilon>0$, there is a neighborhood $V$ of $t$, such that

$$
\left|f(\rho(t))-f(s)-f^{\nabla}(t)(\rho(t)-s)\right| \leq \epsilon|\rho(t)-s|
$$

for all $s \in V$.

A function $f: \mathbb{T} \rightarrow \mathbb{R}$ is said to be left-dense continuous (ld-continuous), provided it is continuous at all left-dense points in $\mathbb{T}$ and its right-sided limits exist (finite) at all right-dense points in $\mathbb{T}$. The set of all ld-continuous functions is denoted by $C_{l d}(\mathbb{T}, \mathbb{R})$.

The next definition is given in $[2,6,7]$.

Definition 2.2. A function $G: \mathbb{T} \rightarrow \mathbb{R}$ is called a nabla antiderivative of $g: \mathbb{T} \rightarrow \mathbb{R}$, provided that $G^{\nabla}(t)=g(t)$ holds for all $t \in \mathbb{T}_{k}$. Then the nabla integral of $g$ is defined by

$$
\int_{a}^{b} g(t) \nabla t=G(b)-G(a) .
$$

Now we present short introduction of diamond $-\alpha$ derivative as given in $[1,19]$.

Let $\mathbb{T}$ be a time scale and $f(t)$ be differentiable on $\mathbb{T}$ in the $\Delta$ and $\nabla$ senses. For $t \in \mathbb{T}_{k}^{k}$, where $\mathbb{T}_{k}^{k}=\mathbb{T}^{k} \cap \mathbb{T}_{k}$, the diamond- $\alpha$ dynamic derivative $f^{\diamond_{\alpha}}(t)$ is defined by

$$
f^{\diamond_{\alpha}}(t)=\alpha f^{\Delta}(t)+(1-\alpha) f^{\nabla}(t), \quad 0 \leq \alpha \leq 1
$$

Thus $f$ is diamond $-\alpha$ differentiable if and only if $f$ is $\Delta$ and $\nabla$ differentiable.

The diamond $-\alpha$ derivative reduces to the standard $\Delta$-derivative for $\alpha=1$, or the standard $\nabla$-derivative for $\alpha=0$. It represents a weighted dynamic derivative for $\alpha \in(0,1)$.

Theorem 2.3 ([19]). Let $f, g: \mathbb{T} \rightarrow \mathbb{R}$ be diamond- $\alpha$ differentiable at $t \in \mathbb{T}$ and we write $f^{\sigma}(t)=f(\sigma(t)), g^{\sigma}(t)=g(\sigma(t)), f^{\rho}(t)=f(\rho(t))$ and $g^{\rho}(t)=g(\rho(t))$. Then 
(i) $f \pm g: \mathbb{T} \rightarrow \mathbb{R}$ is diamond- $\alpha$ differentiable at $t \in \mathbb{T}$, with

$$
(f \pm g)^{\diamond_{\alpha}}(t)=f^{\diamond_{\alpha}}(t) \pm g^{\diamond_{\alpha}}(t) .
$$

(ii) $f g: \mathbb{T} \rightarrow \mathbb{R}$ is diamond- $\alpha$ differentiable at $t \in \mathbb{T}$, with

$$
(f g)^{\diamond_{\alpha}}(t)=f^{\diamond_{\alpha}}(t) g(t)+\alpha f^{\sigma}(t) g^{\Delta}(t)+(1-\alpha) f^{\rho}(t) g^{\nabla}(t) .
$$

(iii) For $g(t) g^{\sigma}(t) g^{\rho}(t) \neq 0, \frac{f}{g}: \mathbb{T} \rightarrow \mathbb{R}$ is diamond- $\alpha$ differentiable at $t \in \mathbb{T}$, with

$$
\left(\frac{f}{g}\right)^{\diamond_{\alpha}}(t)=\frac{f^{\diamond_{\alpha}}(t) g^{\sigma}(t) g^{\rho}(t)-\alpha f^{\sigma}(t) g^{\rho}(t) g^{\Delta}(t)-(1-\alpha) f^{\rho}(t) g^{\sigma}(t) g^{\nabla}(t)}{g(t) g^{\sigma}(t) g^{\rho}(t)} .
$$

Definition $2.4([19])$. Let $a, t \in \mathbb{T}$ and $h: \mathbb{T} \rightarrow \mathbb{R}$. Then the diamond- $\alpha$ integral from $a$ to $t$ of $h$ is defined by

$$
\int_{a}^{t} h(s) \diamond_{\alpha} s=\alpha \int_{a}^{t} h(s) \Delta s+(1-\alpha) \int_{a}^{t} h(s) \nabla s, \quad 0 \leq \alpha \leq 1,
$$

provided that there exist delta and nabla integrals of $h$ on $\mathbb{T}$.

Theorem $2.5([19])$. Let $a, b, t \in \mathbb{T}, c \in \mathbb{R}$. Assume that $f(s)$ and $g(s)$ are $\diamond_{\alpha}-$ integrable functions on $[a, b]_{\mathbb{T}}$. Then

(i) $\int_{a}^{t}[f(s) \pm g(s)] \diamond_{\alpha} s=\int_{a}^{t} f(s) \diamond_{\alpha} s \pm \int_{a}^{t} g(s) \diamond_{\alpha} s$.

(ii) $\int_{a}^{t} c f(s) \diamond_{\alpha} s=c \int_{a}^{t} f(s) \diamond_{\alpha} s$.

(iii) $\int_{a}^{t} f(s) \diamond_{\alpha} s=-\int_{t}^{a} f(s) \diamond_{\alpha} s$.

(iv) $\int_{a}^{t} f(s) \diamond_{\alpha} s=\int_{a}^{b} f(s) \diamond_{\alpha} s+\int_{b}^{t} f(s) \diamond_{\alpha} s$.

(v) $\int_{a}^{a} f(s) \diamond_{\alpha} s=0$.

We need the following results.

Definition 2.6 ([10]). A function $f: \mathbb{T} \rightarrow \mathbb{R}$ is called convex on $I_{\mathbb{T}}=I \cap \mathbb{T}$, where $I$ is an interval of $\mathbb{R}$ (open or closed), if

$$
f(\chi t+(1-\chi) s) \leq \chi f(t)+(1-\chi) f(s),
$$

for all $t, s \in I_{\mathbb{T}}$ and all $\chi \in[0,1]$ such that $\chi t+(1-\chi) s \in I_{\mathbb{T}}$.

The function $f$ is strictly convex on $I_{\mathbb{T}}$ if the inequality (2.1) is strict for distinct $t, s \in I_{\mathbb{T}}$ and $\chi \in(0,1)$.

The function $f$ is concave (respectively, strictly concave) on $I_{\mathbb{T}}$, if $-f$ is convex (respectively, strictly convex). 
Theorem 2.7 ([1]). Let $a, b \in \mathbb{T}$ and $c, d \in \mathbb{R}$. Suppose that $g \in C\left([a, b]_{\mathbb{T}},(c, d)\right)$ and $h \in C\left([a, b]_{\mathbb{T}}, \mathbb{R}\right)$ with $\int_{a}^{b}|h(s)| \diamond_{\alpha} s>0$. If $\Phi \in C((c, d), \mathbb{R})$ is convex, then generalized Jensen's inequality is

$$
\Phi\left(\frac{\int_{a}^{b}|h(s)| g(s) \diamond_{\alpha} s}{\int_{a}^{b}|h(s)| \diamond_{\alpha} s}\right) \leq \frac{\int_{a}^{b}|h(s)| \Phi(g(s)) \diamond_{\alpha} s}{\int_{a}^{b}|h(s)| \diamond_{\alpha} s} .
$$

If $\Phi$ is strictly convex, then the inequality $\leq$ can be replaced by $<$.

Theorem $2.8([16])$. Let $w, f, g \in C\left([a, b]_{\mathbb{T}}, \mathbb{R}\right)$ be $\diamond_{\alpha}$-integrable functions, where $w$, $g \neq 0$. If $\xi \geq 0$, then

$$
\frac{\left(\int_{a}^{b}|w(x)||f(x)| \diamond_{\alpha} x\right)^{\xi+1}}{\left(\int_{a}^{b}|w(x)||g(x)| \diamond_{\alpha} x\right)^{\xi}} \leq \int_{a}^{b} \frac{|w(x)||f(x)|^{\xi+1}}{|g(x)|^{\xi}} \diamond_{\alpha} x .
$$

Inequality (2.3) is called Radon's inequality on time scales and is reversed for $-1<\xi<0$.

\section{Main Results}

In order to present our main results, first we present a simple proof for an extension of Radon's inequality on time scales.

Theorem 3.1. Let $w, f, g \in C\left([a, b]_{\mathbb{T}}, \mathbb{R}\right)$ be $\diamond_{\alpha}$-integrable functions with $\int_{a}^{b}|w(x)| \diamond_{\alpha}$ $x>0$ and $g \neq 0$. If $\beta \geq 2$, then

$$
\left(\int_{a}^{b}|w(x)| \diamond_{\alpha} x\right)^{2-\beta} \frac{\left(\int_{a}^{b}|w(x)||f(x)| \diamond_{\alpha} x\right)^{\beta}}{\int_{a}^{b}|w(x)||g(x)| \diamond_{\alpha} x} \leq \int_{a}^{b} \frac{|w(x)||f(x)|^{\beta}}{|g(x)|} \diamond_{\alpha} x .
$$

Proof. The right-hand side of (3.1) takes the form

$$
\int_{a}^{b} \frac{|w(x)||f(x)|^{\beta}}{|g(x)|} \diamond_{\alpha} x=\int_{a}^{b} \frac{|w(x)||f(x)|^{\beta}}{\left(|g(x)|^{\frac{1}{\beta-1}}\right)^{\beta-1}} \diamond_{\alpha} x
$$

Applying Radon's inequality (2.3), the inequality (3.2) becomes

$$
\int_{a}^{b} \frac{|w(x)||f(x)|^{\beta}}{|g(x)|} \diamond_{\alpha} x \geq \frac{\left(\int_{a}^{b}|w(x)||f(x)| \diamond_{\alpha} x\right)^{\beta}}{\left(\int_{a}^{b}|w(x)||g(x)|^{\frac{1}{\beta-1}} \diamond_{\alpha} x\right)^{\beta-1}}
$$

Note that

$$
\int_{a}^{b}|w(x)||g(x)|^{\frac{1}{\beta-1}} \diamond_{\alpha} x=\int_{a}^{b} \frac{|w(x)||g(x)|^{\frac{1}{\beta-1}}}{1^{\frac{1}{\beta-1}}-1} \diamond_{\alpha} x .
$$


Applying reverse Radon's inequality on right-hand side of (3.4), we get

$$
\int_{a}^{b} \frac{|w(x)||g(x)|^{\frac{1}{\beta-1}}}{1^{\frac{1}{\beta-1}-1}} \diamond_{\alpha} x \leq \frac{\left(\int_{a}^{b}|w(x)||g(x)| \diamond_{\alpha} x\right)^{\frac{1}{\beta-1}}}{\left(\int_{a}^{b}|w(x)| \diamond_{\alpha} x\right)^{\frac{2-\beta}{\beta-1}}} .
$$

From (3.3) and (3.5), we get the proof of the desired result.

Remark 3.2. Let $\alpha=1, \mathbb{T}=\mathbb{Z}, a=1, b=n+1, w \equiv 1, f(k)=x_{k} \in[0,+\infty)$ and $g(k)=y_{k} \in(0,+\infty)$ for $k \in\{1,2, \ldots, n\}$. Then (3.1) reduces to (1.1).

Remark 3.3. Let $\alpha=1, \mathbb{T}=\mathbb{Z}, a=1, b=n+1, w \equiv 1, f(k)=x_{k} \in \mathbb{R}$ and $g(k)=y_{k} \in(0,+\infty)$ for $k \in\{1,2, \ldots, n\}$. If $\beta=2$, then (3.1) reduces to

$$
\frac{\left(\sum_{k=1}^{n} x_{k}\right)^{2}}{\sum_{k=1}^{n} y_{k}} \leq \sum_{k=1}^{n} \frac{x_{k}^{2}}{y_{k}},
$$

which is called Bergström's inequality or Titu Andreescu's inequality, or Engel's inequality in literature as given in $[4,5,8,14]$ with equality if and only if $\frac{x_{1}}{y_{1}}=\frac{x_{2}}{y_{2}}=$ $\ldots=\frac{x_{n}}{y_{n}}$.

The following inequality is called the dynamic weighted power mean inequality on time scales.

Corollary 3.4. Let $w, f \in C\left([a, b]_{\mathbb{T}}, \mathbb{R}\right)$ be $\diamond_{\alpha}$-integrable functions with $\int_{a}^{b}|w(x)| \diamond_{\alpha}$ $x>0$. If $\eta \geq \eta_{1}>0$ and $\eta_{2}=2 \eta$, then

$$
\left(\frac{\int_{a}^{b}|w(x)||f(x)|^{\eta_{1}} \diamond_{\alpha} x}{\int_{a}^{b}|w(x)| \diamond_{\alpha} x}\right)^{\frac{1}{\eta_{1}}} \leq\left(\frac{\int_{a}^{b}|w(x)||f(x)|^{\eta_{2}} \diamond_{\alpha} x}{\int_{a}^{b}|w(x)| \diamond_{\alpha} x}\right)^{\frac{1}{\eta_{2}}} .
$$

Proof. Set $\beta=2\left(\frac{\eta}{\eta_{1}}\right)=\frac{\eta_{2}}{\eta_{1}} \geq 2$ and $g \equiv 1$. The inequality (3.1) reduces to

$$
\left(\int_{a}^{b}|w(x)| \diamond_{\alpha} x\right)^{2-\frac{\eta_{2}}{\eta_{1}}} \frac{\left(\int_{a}^{b}|w(x)||f(x)| \diamond_{\alpha} x\right)^{\frac{\eta_{2}}{\eta_{1}}}}{\int_{a}^{b}|w(x)| \diamond_{\alpha} x} \leq \int_{a}^{b}|w(x)||f(x)|^{\frac{\eta_{2}}{\eta_{1}}} \diamond_{\alpha} x .
$$

Replacing $|f(x)|$ by $|f(x)|^{\eta_{1}}$ and taking power $\frac{1}{\eta_{2}}$ on both sides of (3.8), we get

$$
\begin{gathered}
\left(\int_{a}^{b}|w(x)| \diamond_{\alpha} x\right)^{\frac{1}{\eta_{2}}-\frac{1}{\eta_{1}}}\left(\int_{a}^{b}|w(x)||f(x)|^{\eta_{1}} \diamond_{\alpha} x\right)^{\frac{1}{\eta_{1}}} \\
\leq\left(\int_{a}^{b}|w(x)||f(x)|^{\eta_{2}} \diamond_{\alpha} x\right)^{\frac{1}{\eta_{2}}} .
\end{gathered}
$$

This completes the desired result. 
Remark 3.5. If we set $\alpha=1, \mathbb{T}=\mathbb{Z}, a=1, b=n+1, w(k)=p_{k} \in(0,+\infty)$ and $f(k)=x_{k} \in[0,+\infty)$ for $k \in\{1,2, \ldots, n\}$, then (3.7) reduces to (1.2). Further, if $\sum_{k=1}^{n} p_{k}=1$ and $\eta_{1}=\eta$, then $(1.2)$ reduces to

$$
\left(\sum_{k=1}^{n} p_{k} x_{k}^{\eta_{1}}\right)^{\frac{1}{\eta_{1}}} \leq\left(\sum_{k=1}^{n} p_{k} x_{k}^{2 \eta_{1}}\right)^{\frac{1}{2 \eta_{1}}}
$$

as given in [11].

Now we present Cauchy-Schwarz's inequality on time scales.

Corollary 3.6. Let $w, f, g \in C\left([a, b]_{\mathbb{T}}, \mathbb{R}\right)$ be $\diamond_{\alpha}$-integrable functions. We have:

$$
\begin{gathered}
\left(\int_{a}^{b}|w(x)||f(x) g(x)| \diamond_{\alpha} x\right)^{2} \\
\leq\left(\int_{a}^{b}|w(x)||f(x)|^{2} \diamond_{\alpha} x\right)\left(\int_{a}^{b}|w(x)||g(x)|^{2} \diamond_{\alpha} x\right) .
\end{gathered}
$$

Proof. Setting $\beta=2$ and replacing $|w(x)|$ by $|w(x) g(x)|$ in (3.1), the inequality (3.10) follows.

Remark 3.7. If we set $\alpha=1, \mathbb{T}=\mathbb{Z}, a=1, b=n+1, w \equiv 1, f(k)=x_{k} \in \mathbb{R}$ and $g(k)=y_{k} \in \mathbb{R}$ for $k \in\{1,2, \ldots, n\}$, then (3.10) reduces to (1.3).

Corollary 3.8. Let $w, f \in C\left([a, b]_{\mathbb{T}}, \mathbb{R}-\{0\}\right)$ be $\diamond_{\alpha}$-integrable functions. If $\beta \geq 2$, then

$$
\left(\int_{a}^{b}|w(x)||f(x)| \diamond_{\alpha} x\right)^{\beta} \leq\left(\int_{a}^{b}|w(x)|^{\beta} \diamond_{\alpha} x\right)\left(\int_{a}^{b}|f(x)|^{\frac{\beta}{\beta-1}} \diamond_{\alpha} x\right)^{\beta-1} .
$$

Proof. Let $W, F, G \in C\left([a, b]_{\mathbb{T}}, \mathbb{R}\right)$ be $\diamond_{\alpha}$-integrable functions, neither $W \equiv 0$ nor $G \equiv 0$. If $\beta \geq 2$, then (3.1) takes the form

$$
\left(\int_{a}^{b}|W(x)| \diamond_{\alpha} x\right)^{2-\beta} \frac{\left(\int_{a}^{b}|W(x)||F(x)| \diamond_{\alpha} x\right)^{\beta}}{\int_{a}^{b}|W(x)||G(x)| \diamond_{\alpha} x} \leq \int_{a}^{b} \frac{|W(x)||F(x)|^{\beta}}{|G(x)|} \diamond_{\alpha} x .
$$

Putting $G \equiv 1$ and replacing $|W(x)|$ by $|f(x)|^{\frac{\beta}{\beta-1}}$ and $|F(x)|$ by $|w(x)||f(x)|^{\frac{-1}{\beta-1}}$, we get (3.11). 
Remark 3.9. Let $\alpha=1, \mathbb{T}=\mathbb{Z}, a=1, b=n+1, w(k)=p_{k} \in(0,+\infty)$ and $f(k)=x_{k} \in(0,+\infty)$ for $k \in\{1,2, \ldots, n\}$. If $\beta \geq 2$, then (3.11) reduces to

$$
\left(\sum_{k=1}^{n} p_{k} x_{k}\right)^{\beta} \leq\left(\sum_{k=1}^{n} p_{k}^{\beta}\right)\left(\sum_{k=1}^{n} x_{k}^{\frac{\beta}{\beta-1}}\right)^{\beta-1}
$$

which is symmetric form of Hölder's inequality, as given in [13].

The following result is a generalization of Nesbitt's inequality on time scales.

Theorem 3.10. Let $w, f \in C\left([a, b]_{\mathbb{T}}, \mathbb{R}-\{0\}\right)$ be $\diamond_{\alpha}-$ integrable functions. If $\gamma \geq 1$, $\eta \geq \eta_{1}>0, \eta_{2}=2 \eta, \Omega=\int_{a}^{b}|w(x)||f(x)|^{\eta_{1}} \diamond_{\alpha} x$ and $\Omega>\sup _{x \in[a, b]_{\mathbb{T}}}|f(x)|^{\eta_{1}}$, then

$$
\begin{gathered}
\left(\frac{\int_{a}^{b}|w(x)| \diamond_{\alpha} x}{\left(\int_{a}^{b}|w(x)| \diamond_{\alpha} x-1\right)^{\gamma}}\right)\left(\frac{\Omega}{\int_{a}^{b}|w(x)| \diamond_{\alpha} x}\right)^{\gamma\left(\frac{\eta_{2}}{\eta_{1}}-1\right)} \\
\leq \int_{a}^{b}|w(x)|\left(\frac{|f(x)|^{\eta_{2}}}{\Omega-|f(x)|^{\eta_{1}}}\right)^{\gamma} \diamond_{\alpha} x .
\end{gathered}
$$

Proof. Applying Jensen's inequality for $\gamma>1$, we get

$$
\begin{gathered}
\left(\int_{a}^{b}|w(x)|\left(\frac{|f(x)|^{\eta_{2}}}{\Omega-|f(x)|^{\eta_{1}}}\right) \diamond_{\alpha} x\right)^{\gamma} \\
\leq\left(\int_{a}^{b}|w(x)| \diamond_{\alpha} x\right)^{\gamma-1} \int_{a}^{b}|w(x)|\left(\frac{|f(x)|^{\eta_{2}}}{\Omega-|f(x)|^{\eta_{1}}}\right)^{\gamma} \diamond_{\alpha} x .
\end{gathered}
$$

Now applying Radon's inequality (3.1), we get

$$
\begin{aligned}
& \int_{a}^{b}|w(x)|\left(\frac{|f(x)|^{\eta_{2}}}{\Omega-|f(x)|^{\eta_{1}}}\right) \diamond_{\alpha} x \\
= & \int_{a}^{b}|w(x)|\left(\frac{\left(|f(x)|^{\eta_{1}}\right)^{\frac{\eta_{2}}{\eta_{1}}}}{\Omega-|f(x)|^{\eta_{1}}}\right) \diamond_{\alpha} x \\
\geq & \left(\int_{a}^{b}|w(x)| \diamond_{\alpha} x\right)^{2-\frac{\eta_{2}}{\eta_{1}}} \frac{\left(\int_{a}^{b}|w(x)||f(x)|^{\eta_{1}} \diamond_{\alpha} x\right)^{\frac{\eta_{2}}{\eta_{1}}}}{\int_{a}^{b}|w(x)|\left(\Omega-|f(x)|^{\eta_{1}}\right) \diamond_{\alpha} x} \\
= & \frac{\left(\int_{a}^{b}|w(x)| \diamond_{\alpha} x\right)}{\left(\int_{a}^{b}|w(x)| \diamond_{\alpha} x-1\right)}\left(\frac{\Omega}{\int_{a}^{b}|w(x)| \diamond_{\alpha} x}\right)^{\frac{\eta_{2}}{\eta_{1}}-1} .
\end{aligned}
$$

Thus

$$
\left(\int_{a}^{b}|w(x)|\left(\frac{|f(x)|^{\eta_{2}}}{\Omega-|f(x)|^{\eta_{1}}}\right) \diamond_{\alpha} x\right)^{\gamma} \geq \frac{\left(\int_{a}^{b}|w(x)| \diamond_{\alpha} x\right)^{\gamma}}{\left(\int_{a}^{b}|w(x)| \diamond_{\alpha} x-1\right)^{\gamma}}\left(\frac{\Omega}{\int_{a}^{b}|w(x)| \diamond_{\alpha} x}\right)^{\gamma\left(\frac{\eta_{2}}{\eta_{1}}-1\right)} .
$$


Combining (3.14) and (3.15), we get the desired claim.

Remark 3.11. If we set $\alpha=1, \mathbb{T}=\mathbb{Z}, a=1, b=n+1, w \equiv 1, f(k)=x_{k} \in(0,+\infty)$ for $k \in\{1,2, \ldots, n\}$ and $\sum_{k=1}^{n} x_{k}^{\eta_{1}}>\max _{1 \leq k \leq n} x_{k}^{\eta_{1}}$, then (3.13) reduces to

$$
\left(\frac{n}{(n-1)^{\gamma}}\right)\left(\frac{\sum_{k=1}^{n} x_{k}^{\eta_{1}}}{n}\right)^{\gamma\left(\frac{\eta_{2}}{\eta_{1}}-1\right)} \leq \sum_{k=1}^{n}\left(\frac{x_{k}^{\eta_{2}}}{\sum_{k=1}^{n} x_{k}^{\eta_{1}}-x_{k}^{\eta_{1}}}\right)^{\gamma}
$$

as given in [20].

Further, if we take $\eta_{1}=1, \gamma=1, n=3, x_{1}=x, x_{2}=y$ and $x_{3}=z$, then $(3.16)$ takes the form

$$
\frac{3}{2}\left(\frac{x+y+z}{3}\right)^{\eta_{2}-1} \leq \frac{x^{\eta_{2}}}{y+z}+\frac{y^{\eta_{2}}}{z+x}+\frac{z^{\eta_{2}}}{x+y}
$$

Inequality (3.17) is called the generalized Nesbitt's inequality as given in [20].

The following result is another consequence of Radon's inequality on time scales.

Theorem 3.12. Let $w, f \in C\left([a, b]_{\mathbb{T}}, \mathbb{R}-\{0\}\right)$ be $\diamond_{\alpha}$-integrable functions. If $c_{1} \in$ $[0,+\infty), c_{2}, c_{3}, c_{4} \in(0,+\infty), \gamma, \zeta, \kappa, \lambda \in[1,+\infty)$ and $c_{3}\left(\int_{a}^{b}|w(x)||f(x)| \diamond_{\alpha} x\right)^{\gamma}>$ $c_{4} \sup _{x \in[a, b]_{\mathbb{T}}}|f(x)|^{\gamma}$, then

$$
\begin{gathered}
\frac{\left(c_{1}\left(\int_{a}^{b}|w(x)| \diamond_{\alpha} x\right)^{\kappa}+c_{2}\right)^{\lambda}\left(\int_{a}^{b}|w(x)| \diamond_{\alpha} x\right)^{\gamma \zeta-\kappa \lambda+1}}{\left(c_{3}\left(\int_{a}^{b}|w(x)| \diamond_{\alpha} x\right)^{\gamma}-c_{4}\right)^{\zeta}} \leq\left(\frac{1}{\int_{a}^{b}|w(x)| \diamond_{\alpha} x}\right) \\
\left.\left.\left(\int_{a}^{b}|w(x)||f(x)| \diamond_{\alpha} x\right)^{\kappa \lambda-\gamma \zeta}\right)^{\kappa}+c_{2}|f(x)|^{\kappa} \diamond_{\alpha} x\right\} \\
\left\{\int _ { a } ^ { b } | w ( x ) | \left(c_{1}\left(\int_{a}^{b}|w(x)||f(x)| \diamond_{\alpha} x\right)^{\gamma}\right.\right. \\
\quad\left\{\frac{1}{\left(c_{3}\left(\int_{a}^{b}|w(x)||f(x)| \diamond_{\alpha} x\right)^{\gamma}-c_{4}|f(x)| \gamma\right)^{\zeta}}\right\} \diamond_{\alpha} x .
\end{gathered}
$$

Proof. We obtain the following result by applying Radon's inequality given in (2.3), 
as

$$
\begin{gathered}
\frac{\left(\int_{a}^{b}|w(x)| \diamond_{\alpha} x\right)^{\zeta+1}}{\left\{\int_{a}^{b}|w(x)|\left(c_{3}\left(\int_{a}^{b}|w(x)||f(x)| \diamond_{\alpha} x\right)^{\gamma}-c_{4}|f(x)|^{\gamma}\right) \diamond_{\alpha} x\right\}^{\zeta}} \\
\leq \int_{a}^{b}|w(x)|\left\{\frac{1^{\zeta+1}}{\left(c_{3}\left(\int_{a}^{b}|w(x)||f(x)| \diamond_{\alpha} x\right)^{\gamma}-c_{4}|f(x)|^{\gamma}\right)^{\zeta}}\right\} \diamond_{\alpha} x .
\end{gathered}
$$

Applying (2.2) and (3.19), the right-hand side of (3.18) takes the form

$$
\begin{gathered}
\left(\frac{1}{\int_{a}^{b}|w(x)| \diamond_{\alpha} x}\right)\left\{\int_{a}^{b}|w(x)|\left(c_{1}\left(\int_{a}^{b}|w(x)||f(x)| \diamond_{\alpha} x\right)^{\kappa}+c_{2}|f(x)|^{\kappa}\right)^{\lambda} \diamond_{\alpha} x\right\} \\
\times \int_{a}^{b}|w(x)|\left\{\frac{1}{\left(c_{3}\left(\int_{a}^{b}|w(x)||f(x)| \diamond_{\alpha} x\right)^{\gamma}-c_{4}|f(x)|^{\gamma}\right)^{\zeta}}\right\} \diamond_{\alpha} x \\
\geq\left(\int_{a}^{b}|w(x)| \diamond_{\alpha} x\right)^{\zeta+1-\lambda} \\
\times \frac{\left\{c_{1}\left(\int_{a}^{b}|w(x)| \diamond_{\alpha} x\right)\left(\int_{a}^{b}|w(x)||f(x)| \diamond_{\alpha} x\right)^{\kappa}+c_{2} \int_{a}^{b}|w(x)||f(x)|^{\kappa} \diamond_{\alpha} x\right\}^{\lambda}}{\left\{c_{3}\left(\int_{a}^{b}|w(x)| \diamond_{\alpha} x\right)\left(\int_{a}^{b}|w(x)||f(x)| \diamond_{\alpha} x\right)^{\gamma}-c_{4} \int_{a}^{b}|w(x)||f(x)|^{\gamma} \diamond_{\alpha} x\right\}^{\zeta}} \\
\geq\left(\int_{a}^{b}|w(x)| \diamond_{\alpha} x\right)^{\zeta+1-\lambda} \\
\times \frac{\left\{c_{1}\left(\int_{a}^{b}|w(x)| \diamond_{\alpha} x\right)\left(\int_{a}^{b}|w(x)||f(x)| \diamond_{\alpha} x\right)^{\kappa}+c_{2} \frac{\left(\int_{a}^{b}|w(x)||f(x)| \diamond_{\alpha} x\right)^{\kappa}}{\left(\int_{a}^{b}|w(x)| \diamond_{\alpha} x\right)^{\kappa-1}}\right\}^{\lambda}}{\left\{c_{3}\left(\int_{a}^{b}|w(x)| \diamond_{\alpha} x\right)\left(\int_{a}^{b}|w(x)||f(x)| \diamond_{\alpha} x\right)^{\gamma}-c_{4} \frac{\left(\int_{a}^{b}|w(x)||f(x)| \diamond_{\alpha} x\right)^{\gamma}}{\left(\int_{a}^{b}|w(x)| \diamond_{\alpha} x\right)^{\gamma-1}}\right\}^{\zeta}}
\end{gathered}
$$

Therefore, the inequality (3.18) follows.

Remark 3.13. If we set $\alpha=1, \mathbb{T}=\mathbb{Z}, a=1, b=n+1, w \equiv 1, f(k)=x_{k} \in(0,+\infty)$ for $k \in\{1,2, \ldots, n\}, X_{n}=\sum_{k=1}^{n} x_{k}$ and $c_{3}\left(\sum_{k=1}^{n} x_{k}\right)^{\gamma}>c_{4} \max _{1 \leq k \leq n} x_{k}^{\gamma}$, then (3.18) reduces to

$$
\begin{gathered}
\frac{\left(c_{1} n^{\kappa}+c_{2}\right)^{\lambda}}{\left(c_{3} n^{\gamma}-c_{4}\right)^{\zeta}} n^{\gamma \zeta-\kappa \lambda+1} X_{n}^{\kappa \lambda-\gamma \zeta} \\
\leq \frac{1}{n}\left(\sum_{k=1}^{n}\left(c_{1} X_{n}^{\kappa}+c_{2} x_{k}^{\kappa}\right)^{\lambda}\right) \sum_{k=1}^{n} \frac{1}{\left(c_{3} X_{n}^{\gamma}-c_{4} x_{k}^{\gamma}\right)^{\zeta}},
\end{gathered}
$$


as given in [3].

Corollary 3.14. Let $w, f \in C\left([a, b]_{\mathbb{T}}, \mathbb{R}-\{0\}\right)$ be $\diamond_{\alpha}$-integrable functions. If $c_{1} \in[0,+\infty), c_{2}, c_{3}, c_{4} \in(0,+\infty), \beta \in[2,+\infty)$ and $c_{3} \int_{a}^{b}|w(x)||f(x)| \diamond_{\alpha} x>$ $c_{4} \sup _{x \in[a, b]_{\mathbb{T}}}|f(x)|$, then

$$
\begin{gathered}
\frac{\left(c_{1} \int_{a}^{b}|w(x)| \diamond_{\alpha} x+c_{2}\right)^{\beta}}{c_{3} \int_{a}^{b}|w(x)| \diamond_{\alpha} x-c_{4}}\left(\int_{a}^{b}|w(x)| \diamond_{\alpha} x\right)^{2-\beta}\left(\int_{a}^{b}|w(x)||f(x)| \diamond_{\alpha} x\right)^{\beta-1} \\
\leq \int_{a}^{b}|w(x)|\left\{\frac{\left(c_{1} \int_{a}^{b}|w(x)||f(x)| \diamond_{\alpha} x+c_{2}|f(x)|\right)^{\beta}}{c_{3} \int_{a}^{b}|w(x)||f(x)| \diamond_{\alpha} x-c_{4}|f(x)|}\right\} \diamond_{\alpha} x
\end{gathered}
$$

Proof. By applying (3.1), the right-hand side of (3.21) becomes

$$
\begin{gathered}
\int_{a}^{b}|w(x)|\left\{\frac{\left(c_{1} \int_{a}^{b}|w(x)||f(x)| \diamond_{\alpha} x+c_{2}|f(x)|\right)^{\beta}}{c_{3} \int_{a}^{b}|w(x)||f(x)| \diamond_{\alpha} x-c_{4}|f(x)|}\right\} \diamond_{\alpha} x \\
\geq\left(\int_{a}^{b}|w(x)| \diamond_{\alpha} x\right)^{2-\beta} \frac{\left\{\int_{a}^{b}|w(x)|\left(c_{1} \int_{a}^{b}|w(x)||f(x)| \diamond_{\alpha} x+c_{2}|f(x)|\right) \diamond_{\alpha} x\right\}^{\beta}}{\int_{a}^{b}|w(x)|\left(c_{3} \int_{a}^{b}|w(x)||f(x)| \diamond_{\alpha} x-c_{4}|f(x)|\right) \diamond_{\alpha} x} .
\end{gathered}
$$

Thus inequality (3.21) follows.

Remark 3.15. If we set $\alpha=1, \mathbb{T}=\mathbb{Z}, a=1, b=n+1, w \equiv 1, f(k)=x_{k} \in(0,+\infty)$ for $k \in\{1,2, \ldots, n\}, X_{n}=\sum_{k=1}^{n} x_{k}$ and $c_{3}\left(\sum_{k=1}^{n} x_{k}\right)>c_{4} \max _{1 \leq k \leq n} x_{k}$, then (3.21) reduces to

$$
\frac{\left(c_{1} n+c_{2}\right)^{\beta}}{c_{3} n-c_{4}} n^{2-\beta} X_{n}^{\beta-1} \leq \sum_{k=1}^{n} \frac{\left(c_{1} X_{n}+c_{2} x_{k}\right)^{\beta}}{c_{3} X_{n}-c_{4} x_{k}},
$$

which is similar to an inequality given in [3].

Corollary 3.16. Let $w, f \in C\left([a, b]_{\mathbb{T}}, \mathbb{R}-\{0\}\right)$ be $\diamond_{\alpha}$-integrable functions. If $c_{3}, c_{4} \in$ $(0,+\infty), \beta \in[2,+\infty)$ and $c_{3} \int_{a}^{b}|w(x)||f(x)| \diamond_{\alpha} x>c_{4} \sup _{x \in[a, b]_{\mathbb{T}}}|f(x)|$, then

$$
\begin{aligned}
& \frac{\left(\int_{a}^{b}|w(x)| \diamond_{\alpha} x\right)^{1-\beta}}{c_{3} \int_{a}^{b}|w(x)| \diamond_{\alpha} x-c_{4}}\left(\int_{a}^{b}|w(x)||f(x)| \diamond_{\alpha} x\right)^{\beta} \\
\leq & \int_{a}^{b}|w(x)|\left\{\frac{|f(x)|^{\beta+1}}{c_{3} \int_{a}^{b}|w(x)||f(x)| \diamond_{\alpha} x-c_{4}|f(x)|}\right\} \diamond_{\alpha} x .
\end{aligned}
$$


Proof. Putting $c_{1}=0, c_{2}=1$ and replacing $\beta$ by $\beta+1$ in (3.21), the inequality (3.24) follows.

Remark 3.17. If we set $\alpha=1$, then we get delta versions and if we set $\alpha=0$, then we get nabla versions of diamond $-\alpha$ integral operator inequalities presented in this article.

Also, if we set $\mathbb{T}=\mathbb{Z}$, then we get discrete versions and if we set $\mathbb{T}=\mathbb{R}$, then we get continuous versions of diamond- $\alpha$ integral operator inequalities presented in this article.

\section{Conclusion and Future Work}

There have been recent developments of the theory and applications of dynamic inequalities on time scales. In this research article, we have presented some dynamic inequalities on diamond $-\alpha$ calculus, which is the linear combination of the delta and nabla integrals. Some generalizations and applications of Radon's inequality, Bergström's inequality, Nesbitt's inequality and other dynamic inequalities on time scales are also given in $[17,18]$.

In the future research, we can generalize the well-known inequalities using functional generalization, $n$-tuple diamond- $\alpha$ integral, fractional Riemann-Liouville integral, quantum calculus and $\alpha, \beta$-symmetric quantum calculus.

\section{References}

[1] R.P. Agarwal, D. O'Regan, S.H. Saker, Dynamic Inequalities on Time Scales, Springer International Publishing, Cham, Switzerland 2014.

[2] D. Anderson, J. Bullock, L. Erbe, A. Peterson, H. Tran, Nabla dynamic equations on time scales, Pan-American Mathematical Journal 13 (1) (2003) 1-47.

[3] D.M. Bătineţu-Giurgiu, N. Stanciu, New generalizations and new applications for Nesbitt's inequality, Journal of Science and Arts 12 (2012) no.4 (21) 425430 .

[4] E.F. Beckenbach, R. Bellman, Inequalities, Springer, Berlin, Göttingen and Heidelberg, 1961.

[5] R. Bellman, Notes on matrix theory-IV (An inequality due to Bergström), Amer. Math. Monthly 62 (1955) 172-173.

[6] M. Bohner, A. Peterson, Dynamic Equations on Time Scales, Birkhäuser Boston, Inc., Boston, MA, 2001.

[7] M. Bohner, A. Peterson, Advances in Dynamic Equations on Time Scales, Birkhäuser Boston, Boston, MA, 2003. 
[8] H. Bergström, A triangle inequality for matrices, Den Elfte Skandinaviske Matematikerkongress, Trondheim (1949), Johan Grundt Tanums Forlag, Oslo (1952) 264-267.

[9] Z. Cvetkovski, Inequalities. Theorems, Techniques and Selected Problems, Springer-Verlag Berlin Heidelberg, Heidelberg, 2012.

[10] C. Dinu, Convex functions on time scales, Annals of the University of Craiova, Math. Comp. Sci. Ser. 35 (2008) 87-96.

[11] G.H. Hardy, J.E. Littlewood, G. Pólya, Inequalities, Cambridge University Press, Cambridge, UK, 1934.

[12] S. Hilger, Ein Maßkettenkalkül mit Anwendung auf Zentrumsmannigfaltigkeiten, Ph.D. Thesis, Universität Würzburg, 1988.

[13] L. Maligranda, Why Hölder's inequality should be called Rogers' inequality, Mathematical Inequalities \& Applications 1 (1) (1998) 69-83.

[14] D.S. Mitrinović, Analytic Inequalities, Springer-Verlag, Berlin, 1970.

[15] D.S. Mitrinović, J.E. Pečarić, A.M. Fink, Classical and New Inequalities in Analysis, Mathematics and Its Applicatins (East European Series), vol. 61, Kluwer Academic Publishers, Dordrecht, 1993.

[16] M.J.S. Sahir, Hybridization of classical inequalities with equivalent dynamic inequalities on time scale calculus, The Teaching of Mathematics, XXI (2018) no.1 $38-52$.

[17] M.J.S. Sahir, Formation of versions of some dynamic inequalities unified on time scale calculus, Ural Mathematical Journal 4 (2) (2018) 88-98.

[18] M.J.S. Sahir, Symmetry of classical and extended dynamic inequalities unified on time scale calculus, Turkish J. Ineq. 2 (2) (2018) 11-22.

[19] Q. Sheng, M. Fadag, J. Henderson, J.M. Davis, An exploration of combined dynamic derivatives on time scales and their applications, Nonlinear Anal. Real World Appl. 7 (3) (2006) 395-413.

[20] F. Wei, S. Wu, Generalizations and analogues of the Nesbitt's inequality, Octogon Mathematical Magazine 17 (1) (2009) 215-220.

[21] S. Wu, An exponential generalization of a Radon inequality, J. Huaqiao Univ. Nat. Sci. Ed. 24 (1) (2003) 109-112.

[22] S. Wu, A result on extending Radon's inequality and its application, J. Guizhou Univ. Nat. Sci. Ed. 22 (1) (2004) 1-4.

[23] S. Wu, A new generalization of the Radon inequality, Math. Practice Theory 35 (9) (2005) 134-139. 
[24] S. Wu, A class of new Radon type inequalities and their applications, Math. Practice Theory 36 (3) (2006) 217-224.

DOI: $10.7862 / \mathrm{rf.2020.10}$

Muhammad Jibril Shahab Sahir

email: jibrielshahab@gmail.com

ORCID: 0000-0002-7854-8266

University of Sargodha

Sub-Campus Bhakkar

Bhakkar

PAKISTAN

Received 04.04.2020

Accepted 17.08.2020 